Experimental Study

\title{
Using first bout effect to study the mechanisms underlying eccentric exercise induced force loss
}

\author{
Orawan Prasartwuth ${ }^{\mathrm{a}, ~}{ }^{*}$, Roongtip Suteebut ${ }^{\mathrm{a}}$, Jitapa Chawawisuttikool a , Utku S. Yavuz ${ }^{\mathrm{b}}$, \\ Kemal S. Turker ${ }^{c}$ \\ a Department of Physical Therapy, Faculty of Associated Medical Sciences, Chiang Mai University, Thailand \\ ${ }^{\mathrm{b}}$ Department of Neurorehabilitation Engineering, Bernstein Focus Neurotechnology Gottingen, Bernstein Centre for Computational Neuroscience, Germany \\ ${ }^{\mathrm{c}}$ Koc University School of Medicine, Sariyer, Istanbul, Turkey
}

\section{A R T I C L E I N F O}

\section{Article history:}

Received 16 August 2017

Received in revised form

5 September 2017

Accepted 21 November 2017

\section{Keywords:}

Voluntary activation

Muscle damage

Repeated bout of eccentric exercise

Neural adaptations and muscular

adaptations

\begin{abstract}
A B S T R A C T
Introduction: The first bout of eccentric exercise is known to have a protective effect on the consequent bouts. This effect is still disputable as it is not known whether it protects muscle damage by reducing force production or by improving force recovery in the healing process. The underlying mechanisms of this protective effect have not been fully understood.

Objectives: To determine the mechanisms of this protective effect, three different loads were used for the first eccentric bout. This was done to investigate whether the protective effect is related to the size of the load in the first bout. To determine the neural adaptations, voluntary activation was assessed and to determine the muscular adaptations, the resting twitch was measured.

Method: Thirty healthy participants were selectively allocated into three groups (low-, moderate- and high-load group) to match for maximal voluntary contraction (MVC) $(n=10$ per group). Participants in each group performed only one of the three sets of ten eccentric (ECC) exercises of the elbow flexors (10\%, 20\% and $40 \%$ of MVC) as their first eccentric bout. The second bout of eccentric exercise was performed two weeks later and was identical for all the three groups, i.e., $40 \%$ ECC.

Results: The results showed that for the first bout, MVC, voluntary activation and the resting twitch displayed significant $(\mathrm{p}<0.0001)$ interaction (group $\mathrm{x}$ time). This was not the case however for the second bout as there was no significant (group $\mathrm{x}$ time) interaction in all outcome variables immediately after exercise. When the first and second bouts were compared, it was found that the high-load group had faster recovery in MVC at day 1 and 4 corresponding to voluntary activation and only at day 4 corresponding to the resting twitch.

Conclusions: In this study, it was found that high-load exercise aids fast recovery either via neural or muscular adaptations.
\end{abstract}

() 2017 Elsevier Ltd. All rights reserved.

\section{Introduction}

Maximal force production or maximal voluntary isometric contraction is used as the final outcome in studies of human physiology where muscle strength is assessed. To assess the central fatigue, electrically induced twitch during maximal voluntarilyexerted force (twitch interpolated method) is performed and used to calculate the voluntary activation. Electrically induced

\footnotetext{
* Corresponding author. Department of Physical Therapy, Faculty of Associated Medical Sciences, Chiang Mai University, Chiang Mai, Thailand.

E-mail address: orawan.pr@cmu.ac.th (O. Prasartwuth).
}

twitch in relaxed muscle bypasses the neural drive (resting twitch) can also be used to calculate voluntary activation (Herbert and Gandevia, 1999). Although this method has some limitations (e.g. single twitch) and is still controversial (methodological discrepancy), it has some advantages over the conventional electromyography (EMG) since it gives insight about neural drive to a muscle without the problems of EMG at a high level of force (Keenan et al., 2005).

The potential mechanisms underlying exercise-induced prolonged strength loss have been reported to be either neuronal control or muscular damage (Prasartwuth et al., 2005). However, exercise or training can improve the magnitude of strength loss. This phenomenon is called "repeated bout effect" (RBE) of eccentric 
exercise. As full activation in eccentric exercise can be reported in athletes (Amiridis et al., 1996; Babault et al., 2001), it is possible to study the underlying mechanisms of repeated bout effects regarding to neural or muscular adaptations during force recovery.

As regard motor unit recruitment, high-threshold motor units were selectively recruited in soleus and gastrocnemius muscles during eccentric contraction (Nardone et al., 1989) and this mechanism is believed to be responsible for high force generation capacity. However, low-threshold motor units were activated in the first dorsal interosseous muscles during eccentric contraction (Howell et al., 1995). This discrepancy may be related to different muscle sites and functions. In addition, discharge rate of motor units reduced markedly during lifting and lowering of a load. With twitch interpolation, an impaired voluntary activation of the elbow flexor in the early stage and damage to the muscle fibers in the later stage has been confirmed as a result of eccentric exercise (Prasartwuth et al., 2005).

Based on the potential sites of neural control (e.g. motor cortex, thalamus and spinal cord), the silent period after the motor evoked potential (MEP) is shorter during eccentric contractions and is followed by intracortical facilitation (Duclay et al., 2011). Also there is evidence to indicate that enhanced intracortical and interhemispheric connections are involved in eccentric contractions (Fang et al., 2004; Fang et al., 2001). In contrast, EMG recordings displayed less even high force production during eccentric exercise compared to concentric and isometric exercises (Aagaard et al., 2000; Loscher and Nordlund, 2002) which is thought to be a compensation for spinal inhibition (Gruber et al., 2009). Therefore, neuronal control could play an important role in protecting against muscle damage. When performing this exercise again for RBE, the protective phenomenon could be illustrated as neural adaptation. However, a previous research using the trained-electrically induced twitch interpolation technique reported that voluntary activation remained the same as pre-exercise level ( $94 \%)$ up to day 1 in knee extensors at the same angle, thus suggesting no neural adaptations as a result of RBE (Kamandulis et al., 2010). To determine the neural adaptation as a result of RBE, this research aimed to investigate whether the underlying mechanisms in neuromuscular adaptations help to protect muscle damage or not. In addition, this study examined the relation between the magnitude of the first bout and the neuromuscular adaptations where repeated bouts exists. To answer the latter question, three different loads of the first eccentric bout were designed to use and investigate their effects on high load repeated eccentric exercise. Previous research suggests that the magnitude of the repeated bout depends on the magnitude of exercise load in the first bout, i.e. the more impaired voluntary activation is in the first bout, the greater the protective effect in the repeated bouts. This knowledge is important as eccentric exercise causes significant force loss, pain and stiffness. For unaccustomed persons, patients or athletes that perform the eccentric exercise again, it is possible to reduce the consequences by choosing an appropriate load.

\section{Method}

\subsection{Participants and study design}

Thirty healthy male and female volunteers who had not performed regular resistance training participated in this study. All participants gave written informed consent prior to the study, which was approved by the Institute Ethics Committee and complied with the Helsinki Declaration. The participants were placed into one of the three groups ( $n=10$ per group) based on the baseline maximal voluntary isometric contraction torque (MVC) of the elbow flexors at an elbow joint angle $90^{\circ}$ to match the average
MVC amongst the groups. The three groups were low-load (10\% ECC), moderate-load (20\% ECC) and high-load (40\% ECC), and the participants in each group performed the first eccentric exercise bout at the chosen intensity, but all groups performed the same eccentric exercise (40\% ECC) two weeks after the first bout. No significant difference in the baseline MVC (164.3 (61.7) N), age (27.1 (5.8) y), height (158 (16.8) cm) and body mass (60.3 (19.3) $\mathrm{kg}$ ) were evident across the groups. Each group consisted of both genders and the number of women for the 10\% ECC, 20\% ECC and 40\% ECC were eight, seven and eight, respectively. All participants were asked to refrain from unaccustomed exercise or vigorous physical activity, and not to take any anti-inflammatory drugs or nutritional supplements during the experimental period. The dependent variables included maximal voluntary contraction, voluntary activation and the resting twitch. Measurements were taken before, immediately after, 1 and 4 days after exercise for the first and second bouts. Changes in these variables over time were compared amongst the groups and bouts.

\subsection{Eccentric exercise}

Participants performed eccentric exercise of the elbow flexors with the non-dominant arm using a dumbbell adjusted to $10 \%, 20 \%$ or $40 \%$ of individual's MVC at an elbow angle of $90^{\circ}$ for the first bout, and the $40 \%$ load exercise for the second bout. The exercise consisted of three sets ten repetitions with a 2 min rest between sets. The participants were instructed to lower the dumbbell from an elbow flexed $\sim 90^{\circ}$ to an elbow extended position $\left(0^{\circ}\right)$ in $4-5 \mathrm{~s}$, keeping the velocity as constant as possible in metronome rhythm. After each eccentric contraction, the investigator lifted the load up while participants relaxed and the arm was passively returned to the starting position.

\subsection{Maximal isometric flexion torques}

For maximal voluntary contraction of elbow flexor muscle group, participants sat with the shoulder and elbow joint held at $90^{\circ}$ flexion in an arm bar attached with a force gauge (2KN, A\&D CO, Ltd, Japan) connected to a PowerLab. This arm bar was used to measure isometric flexion torque at $90^{\circ}$ elbow flexion. Verbal encouragement was provided during MVC measurements. Three measurements of maximal effort were made with a 5-s rest between trials and the maximal value of the three was used for further analysis.

\subsection{Motor nerve stimulation}

For stimulation of the motor nerve, single electrical stimulus (100 $\mu$ s duration, constant current) was delivered to nerve fibers innervating the biceps brachii via a surface cathode located midway between the anterior edge of the deltoid and the elbow crease and a surface anode positioned over the distal biceps tendon using a Digitimer. The stimulation intensity was set at $10 \%$ above the level required to produce a resting twitch of maximal amplitude. The stimulus intensity was set at each measurement session. The sites of stimulation were marked on the skin to ensure consistent placement for all measurements. During the electrical stimulation of the motor nerve, any increment in elbow flexion torque evoked during a MVC was expressed as a fraction of the amplitude of the maximal response evoked by the same stimulus in the relaxed muscle immediately after an MVC ("resting twitch; RT"). Voluntary activation was then quantified as a percentage using the following formula (see Herbert and Gandevia, 1999): 
Voluntary activation $(\%)=(1$-superimposed twitch/resting twitch) $\mathrm{x} 100$

\subsection{Statistical analysis}

Changes in the dependent variables over time were compared amongst the groups for the first eccentric bout and the second eccentric bout separately by a two-way repeated measure of Analysis of Variance (ANOVA). The ANOVA was performed for both raw and normalized data for all variables except for voluntary activation. For the ANOVA using normalized data, pre-exercise values (100 for MVC and RT) were excluded. The ANOVA also compared the first $40 \%$ bout performed by the $40 \%$ ECC group and the second eccentric exercise bout performed by all groups in which the $40 \%$ load was used to examine whether the first exercise bout conferred any protective effect. When the ANOVA showed a significant interaction (group $\mathrm{x}$ time) effect, a Tukey's HSD test was employed as post hoc analysis to locate the time points of significant differences between groups. One-way ANOVA was also used to compare groups at each time. Statistical significance was set at $\mathrm{p}<0.05$. The results are presented as means (SD).

\section{Results}

\subsection{First eccentric exercise}

\subsubsection{Deficit in force production}

For the first bout, MVC in low-load group decreased by $7.12 \%$ (from 167.9 (63.8) to 153.9 (53.1) N), MVC in moderate-load group decreased by $12.76 \%$ (from 170.0 (59.4) to 147.8 (54.7) N) and MVC in high-load group decreased by $45.61 \%$ (from 155.0 (61.8) to 81.4 $(31.5) \mathrm{N}$ ) of the pre-exercise value immediately after eccentric exercise and recovered back to normal at day 4 only for the low-load and moderate-load groups. In contrast, with the high-load group, it only recovered to $76.25 \%$ of the initial value at day 4 (Fig. 1, upper panel, left graph). When compared between groups, changes in raw and normalized maximal voluntary contraction torques in all groups over time (group $x$ time) were significantly $(\mathrm{p}<0.0001)$ different. Post hoc analysis found significantly ( $\mathrm{p}<0.0001)$ different between low- and high-load groups, low- and moderateload groups, and moderate- and high-load groups.

\subsubsection{Voluntary activation}

With the low-load group, voluntary activation remained the same after the exercise up to 4 day. While the moderate-load group, voluntary activation decreased slightly by $6.82 \%$ (from $99.26(0.84)$ to 92.44 (4.79) \%) immediately after exercise and returned back to normal at day 4 . With the high-load group, voluntary activation markedly decreased by $32.62 \%$ (from 96.83 (2.64) to 64.21 (18.35) $\%$ ) immediately after exercise and gradually recovered to 91.92 (6.06) \% at day 4 (Fig. 1, middle pane, left graph). When compared between groups, changes in voluntary activation in all groups over time (group $x$ time) were significantly $(\mathrm{p}<0.001)$ different. There were significant $(\mathrm{p}<0.001)$ differences between low- and highload groups, between low- and moderate-load groups, and also between moderate- and high-load groups.

\subsubsection{Resting twitch}

The resting twitches produced by motor nerve stimulation decreased by $26.7 \%$ (from $12.12(6.21)$ to $8.80(4.27) \mathrm{N}$ ), $63.91 \%$ (from $12.36(3.14)$ to $4.41(1.80) \mathrm{N}$ ) and $85.68 \%$ (from 12.97 (6.26) to $1.98(2.40) \mathrm{N}$ ) of their pre-exercise values with the low-, moderateand high-load groups of eccentric exercise respectively. They then gradually recovered to $87.95 \%, 73.45 \%$ and $50.12 \%$ of their pre- exercise values at day 4 with the low-, moderate- and high-load groups respectively (Fig. 1, lower panel, left graph). Changes in the resting twitches in all groups over time (group $x$ time) were significantly ( $\mathrm{p}=0.0001$ ) different. When comparing the resting twitches between low- and moderate-load groups, and between low- and high-load groups, there were significant differences ( $p=0.01, p=0.0001$ respectively). However, there was no significant difference $(\mathrm{p}=0.095)$ between moderate- and high-load groups.

\subsection{Second eccentric exercise}

\subsubsection{Deficit in force production}

When each group performed eccentric exercise with the same high-load (40\% of MVC) after two weeks, immediately after the exercise, the maximal voluntary contraction forces in all groups decreased by $37.99-47.7 \%$ of their initial values. At the 4 day, the maximal voluntary torques in low- and moderate-load groups gradually recovered to $\sim 90 \%$ of their pre-exercise values. Interestingly, the maximal voluntary torques in high-load group returned back to normal (Fig. 1, upper panel, right graph). When the second bout in all groups was compared with the first eccentric using repeated measures ANOVA, the normalized data showed a significant main effect for time $(\mathrm{F}=145.723, \mathrm{p}=0.0001)$. Comparison between groups also showed significant differences $(F=3.885$, $\mathrm{p}=0.017$ ). There was also a significant interaction effect (group $\mathrm{x}$ time) $(\mathrm{F}=3.250, \mathrm{p}=0.007)$. One-way ANOVA found significant differences between high-load group and the baseline at day 1 and $4(\mathrm{p}=0.014, \mathrm{p}=0.003$, respectively) (Fig. 1 , upper panel, right graph).

\subsubsection{Voluntary activation}

All groups showed similar changes and recovery patterns as the exercise load was equal for each group (40\% of MVC). Voluntary activation dropped in similar amounts for the groups after exercise (71.56-78.56\%). At day 4, all groups recovered back to pre-exercise value ( $96 \%$ ) (Fig. 1, middle panel, right graph). When comparing the second bout in all groups with the first eccentric bout using repeated measures ANOVA, the voluntary activation showed a significant main effect for time $(F=40.99, \mathrm{p}=0.0001)$. Comparison between groups showed no significant differences $(F=2.313$, $\mathrm{p}=0.093$ ). There was also no significant interaction effect (group $\mathrm{x}$ time) $(\mathrm{F}=0.638, \mathrm{p}=0.762)$. In the absence of a significant interaction effect, one-way ANOVA was applied, which revealed that there were significant differences between high-load group and the baseline at day 1 and 4 (Fig. 1, middle panel, right graph).

\subsubsection{Resting twitch}

All groups showed similar changes after exercise and similar recovery rate. With normalized data, the resting twitches decreased by $74-86 \%$ (from $\sim 13$ to $\sim 2 \mathrm{~N}$ ) after exercise and return back by $\sim 26 \%$ (from $\sim 13$ to $\sim 9 \mathrm{~N}$ ) at day 4 (Fig. 1, lower panel, right graph). When the second bout in all groups was compared with the first bout using repeated measures ANOVA, the raw and normalized data showed a significant main effect for time $(F=117.27$, $\mathrm{p}=0.0001$ ). There were no significant differences between groups $(\mathrm{F}=1.103, \mathrm{p}=0.367)$. There was also no significant interaction effect (group $x$ time) $(F=0.756, p=0.526)$. In the absence of a significant interaction effect, one-way ANOVA was applied, which revealed that there was only a significant difference between highload group and the baseline at day 4 (Fig. 1, lower panel, right graph). 

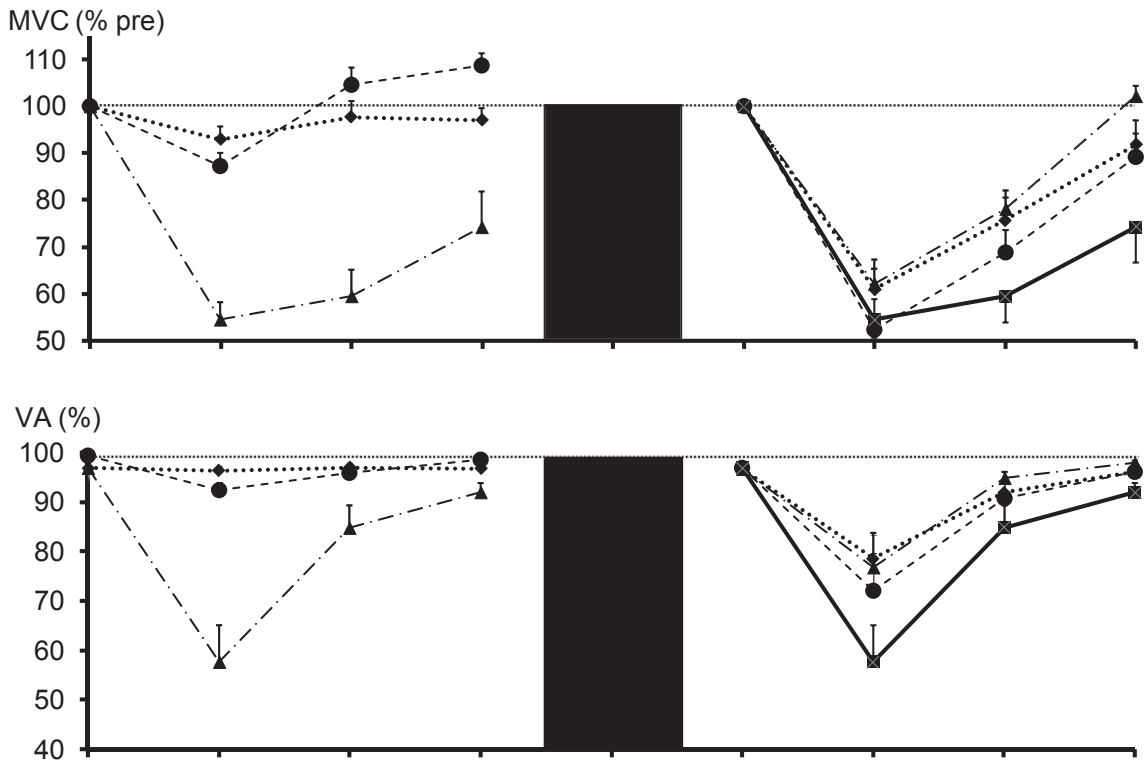

RT (\% pre)

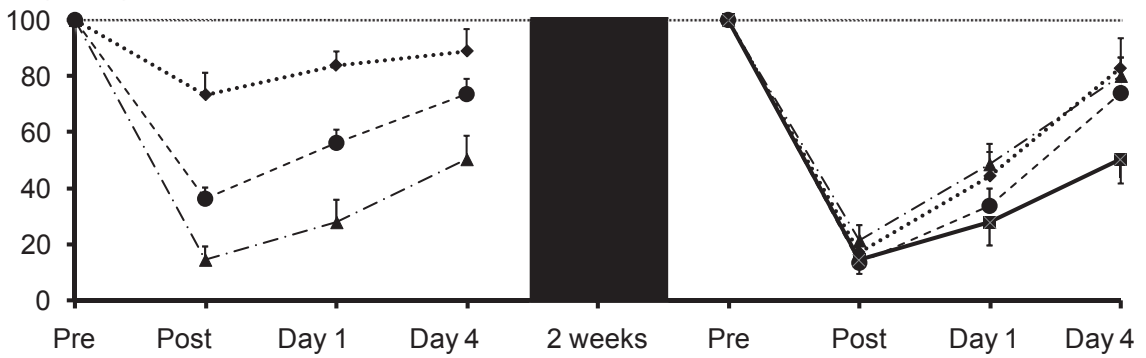

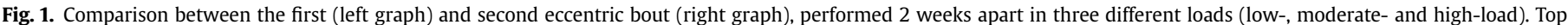
panel represents changes in MVC. Middle panel represents changes in voluntary activation. Lower panel represents changes in the resting twitch.

\section{Discussion}

There are two main findings in this study. Firstly, unlike our hypothesis, we did not observe any protective effect of the first eccentric exercise because the force decreased immediately after the exercise during the second bout at the same amount as in the first bout indicating that the force loss depends on magnitude of current load rather than the prior attempt. Secondly, all three groups showed better force recoveries in the second bout than the baseline (the same load of the first eccentric bout), however, only high-load group reported significantly enhanced recovery.

\subsection{First bout of eccentric exercise: MVC}

Immediately after exercise, low-, moderate- and high-load groups showed the decreases in maximal forces by $7.12 \%, 12.76 \%$ and $45.61 \%$ of their pre-exercise values, respectively. These changes corresponded with impaired voluntary activation by $0 \%, 6.82 \%$ and $32.62 \%$, respectively. These results suggest that impairments in voluntary activation played a role in moderate- and high-load groups. Although the present results confirm previous study with regard to high-load group (Prasartwuth et al., 2005). Kamandulis et al. (2010) has contrary results suggesting that voluntary activation remained the same as the pre-exercise level ( $94 \%)$ up to day 1 in knee extensors at the same angle. The discrepancies in these results need to be interpreted with caution. Possible explanation might be related to different types of stimuli (single and tetanic) and recruitment of synergist and/or antagonist muscles during tetanic stimulation, hence disguising the impaired voluntary activation.

Corresponding to force loss, the amplitude of the resting twitch decreased by $\sim 27 \%, \sim 64 \%$ and $\sim 86 \%$ of their pre-exercise values, respectively. These changes in high-load group were found to be in the line with previous studies (Prasartwuth et al 2005, 2006). Using the same twitch interpolation technique to assess the resting twitch, force loss by $\sim 35-40 \%$ was associated with the decrease in the resting twitch by $\sim 70-85 \%$. However, with different frequency i.e. tetanic stimulation, force loss by $\sim 42 \%$ was associated with the decrease in the resting twitch by $44 \%$ (Kamandulis et al., 2010). Less decrease in the resting twitch from tetanic stimulation could be due to an alteration of the relationship between local intracellular $\mathrm{Ca}^{++}$and force (Gandevia, 2009) and could possibly relate to low- and high-frequency fatigue (Jones, 1996). 


\subsection{Second bout of eccentric exercise}

Immediately after exercise, force deficits were of similar magnitude in all three groups as compared to the baseline. Our results were consistent with the findings of most of the previous studies of this topic (Chen et al 2007, 2009; Kamandulis et al., 2010; Nosaka and Newton, 2002; Nosaka et al., 2001a, 2001b; Paschalis et al., 2008). These findings negated the protective effect of using different loads in the first bout on maximal voluntary torques immediately after exercise. Force deficits depend primarily on the extent of performed current load but they do benefit from exercise in prior bout; in other word, there is no effect of damage prevention (Yu et al 2003, 2004). Therefore, force deficits could primarily be load-dependent immediately after exercise.

At day 1 and 4, all three groups showed better force recovery than the baseline (with the same load of the first eccentric bout) but only the high-load group reported significantly enhanced recovery. There are two possibilities to explain these results. First, the first bout of high-load eccentric exercise could be the best choice to train the athletes even though it causes more muscle damage and impaired voluntary activation to the muscle fibers. Second, it may relate to load specificity, which means the adaptation ability may depend on the exact load given before.

Force output depends on voluntary activation (neural drive to the muscle) and the resting twitch (the contractile element). With reference to voluntary activation, force recovery corresponded with voluntary activation only in the high-load group. At day 1 , maximal force significantly recovered to $\sim 80 \%$ of pre-exercise level while voluntary activation significantly improved to $~ 93 \%$. At day 4 , maximal force significantly recovered back to pre-exercise level and voluntary activation also recovered back to pre-exercise level. These imply that the ability of the neural system to recover or for neural adaptation to take place is demonstrated in the early phase of high-load eccentric exercise. Fast recovery of force loss might be related to enhance neural drive to the muscle fibers. Underlying mechanisms in neural adaptations could occur at the level of motor cortex, spinal cord and/or neuromuscular junction (e.g. increased descending drive, elevated motoneuron excitability and decreased presynaptic inhibition).

In addition, only at day 4 in the high-load group, the resting twitch was significantly depressed at $\sim 78 \%$ of pre-exercise level. Possible explanations might relate to muscular adaptations which could be an increase in sarcomere in series and/or in parallel, Z-line and/or titin remodeling process recovery resulting in an increase in fascicle length and an alter in optimal angle during maximal contraction as well as an increase in pennation angle (Hedayatpour and Falla, 2015).

These findings suggest that neural adaptations could play a primary role in the early phase of the second bout, following which muscular adaptations could then take place with neural adaptations during the force recovery. Only one previous study (Kamandulis et al., 2010) investigated changes in voluntary activation and reported the opposite findings. This discrepancy could be due to different techniques used. We used single twitch whereas in the previous study mentioned above, tetanic stimuli was used. In addition, different methods of calculations were used to assess neural contribution, specifically, we used voluntary activation but the previous study used central activation ratio (CAR) and voluntary activation (VA). In that study, there were no significant changes in CAR and VA, suggesting no neural adaptations, even in the first eccentric bout. Thus, in the second bout, it is not surprising that repeated bout is not associated with changes in voluntary activation. Possible explanation for the different techniques being used (i.e. paired twitch or tetanic stimulation) could be that the magnitude of resting twitch might only involve the contractile, non-contractile elements and/or synergists as well as antagonists.

A limitation of this study could be that single-pulse electrical stimulus might be insufficient to stimulate the resting twitch after eccentric exercise. However, tetanic stimuli could recruit the motor units either in agonist and antagonist muscles. Therefore, paired pulse electrical stimulation could be a good choice to assess voluntary activation after eccentric exercise in future research.

Based on the results, it appears that our body directly responds to the applied load as can be seen by the decreased MVC in the same amount in all groups immediately after the exercise in the second bout. These imply that there is no protective effect in RBE. However, the influence of the high-load eccentric exercise in the first bout assists to improve MVC in day 1 and 4 in the second bout. Better recovery in force is found either in the neural adaptation earlier or in the muscular adaptation later on. This can be applied using the high-load exercise in the first bout in the training even though we know that it causes more force loss.

In summary, the repeated eccentric effect was demonstrated only in the high-load group which could imply a load-specific adaptation. We did not observe any protective effect of the first eccentric exercise because the force decreased immediately after the exercise thus indicating that the force loss depends on magnitude of current load rather than the prior attempt. However, significant recovery in the force generating capacity was found only in the high-load group and this corresponded with the improvement of voluntary activation in day 1 and 4 .

\section{Acknowledgments}

We wish to acknowledge support from Thailand Research Fund and Commission on Higher Education (MRG5280246) and travelling Research Fund from The Research Administration Center, Chiang Mai University.

\section{References}

Aagaard, P., Simonsen, E.B., Andersen, J.L., Magnusson, S.P., Halkjær-Kristensen, J., Dyhre-Poulsen, P., 2000. Neural inhibition during maximal eccentric and concentric quadriceps contraction: effects of resistance training. J. Appl. Physiology 89, 2249-2257.

Amiridis, I.G., Martin, A., Morlon, B., Martin, L., Cometti, G., Pousson, M., van Hoecke, J., 1996. Co-activation and tension-regulating phenomena during isokinetic knee extension in sedentary and highly skilled humans. Eur. J. Appl. Physiology Occup. Physiology 73, 149-156.

Babault, N., Pousson, M., Ballay, Y., Van Hoecke, J., 2001. Activation of human quadriceps femoris during isometric, concentric, and eccentric contractions. J. Appl. Physiology 91, 2628-2634.

Chen, T.C., Chen, H.L., Lin, M.J., Wu, C.J., Nosaka, K., 2009. Muscle damage responses of the elbow flexors to four maximal eccentric exercise bouts performed every 4 weeks. Eur. J. Appl. Physiology 106, 267-275.

Chen, T.C., Nosaka, K., Sacco, P., 2007. Intensity of eccentric exercise, shift of optimum angle, and the magnitude of repeated-bout effect. J. Appl. Physiology 102, 992-999.

Duclay, J., Pasquet, B., Martin, A., Duchateau, J., 2011. Specific modulation of corticospinal and spinal excitabilities during maximal voluntary isometric, shortening and lengthening contractions in synergist muscles. J. Physiology 589, $2901-2916$.

Fang, Y., Siemionow, V., Sahgal, V., Xiong, F., Yue, G.H., 2001. Greater movementrelated cortical potential during human eccentric versus concentric muscle contractions. J. Neurophysiology 86, 1764-1772.

Fang, Y., Siemionow, V., Sahgal, V., Xiong, F., Yue, G.H., 2004. Distinct brain activation patterns for human maximal voluntary eccentric and concentric muscle actions. Brain Res. 1023, 200-212.

Gandevia, S.C., 2009. Twitch interpolation a valid measure with misinterpreted meaning. J. Appl. Physiology 107 (363-364), 367-368.

Gruber, M., Linnamo, V., Strojnik, V., Rantalainen, T., Avela, J., 2009. Excitability at the motoneuron pool and motor cortex is specifically modulated in lengthening compared to isometric contractions. J. Neurophysiology 101, 2030-2040.

Hedayatpour, N., Falla, D., 2015. 2015 physiological and neural adaptations to eccentric exercise: mechanisms and considerations for training. BioMed Res. Int. 193741.

Herbert, R.D., Gandevia, S.C., 1999. Twitch interpolation in human muscles: mechanisms and implications for measurement of voluntary activation. J. Neurophysiology 82, 2271-2283. 
Howell, J.N., Fuglevand, A.J., Walsh, M.L., Bigland-Ritchie, B., 1995. Motor unit activity during isometric and concentric-eccentric contractions of the human first dorsal interosseus muscle. J. Neurophysiology 74, 901-904.

Jones, D.A., 1996. High-and low-frequency fatigue revisited. Acta physiol. Scand 156, 265-270.

Kamandulis, S., Skurvydas, A., Brazaitis, M., Skikas, L., Duchateau, J., 2010. The repeated bout effect of eccentric exercise is not associated with changes in voluntary activation. Eur. J. Appl. Physiology 108, 1065-1074.

Keenan, K.G., Farina, D., Maluf, K.S., Merletti, R., Enoka, R.M., 2005. Influence of amplitude cancellation on the simulated surface electromyogram. J. Appl. Physiology 98, 120-131.

Loscher, W.N., Nordlund, M.M., 2002. Central fatigue and motor cortical excitability during repeated shortening and lengthening actions. Muscle \& Nerve 25, $864-872$.

Nardone, A., Romano, C., Schieppati, M., 1989. Selective recruitment of highthreshold human motor units during voluntary isotonic lengthening of active muscles. J. Physiology 409, 451-471.

Nosaka, K., Newton, M., 2002. Repeated eccentric exercise bouts do not exacerbate muscle damage and repair. J. Strength Cond. Res. 16, 117-122.

Nosaka, K., Sakamoto, K., Newton, M., Sacco, P., 2001a. How long does the protective effect on eccentric exercise-induced muscle damage last? Med. Sci. Sports Exerc. 33, 1490-1495.

Nosaka, K., Sakamoto, K., Newton, M., Sacco, P., 2001b. The repeated bout effect of reduced-load eccentric exercise on elbow flexor muscle damage. Eur. J. Appl. Physiology 85, 34-40.

Paschalis, V., Nikolaidis, M.G., Giakas, G., Jamurtas, A.Z., Owolabi, E.O., Koutedakis, Y., 2008. Position sense and reaction angle after eccentric exercise: the repeated bout effect. Eur. J. Appl. Physiology 103, 9-18.

Prasartwuth, O., Allen, T.J., Butler, J.E., Gandevia, S.C., Taylor, J.L., 2006. Lengthdependent changes in voluntary activation, maximum voluntary torque and twitch responses after eccentric damage in humans. J. Physiology 571, 243-252.

Prasartwuth, O., Taylor, J.L., Gandevia, S.C., 2005. Maximal force, voluntary activation and muscle soreness after eccentric damage to human elbow flexor muscles. J. Physiology 567, 337-348.

Yu, J.G., Carlsson, L., Thornell, L.E., 2004. Evidence for myofibril remodeling as opposed to myofibril damage in human muscles with DOMS: an ultrastructural and immunoelectron microscopic study. Histochem. Cell Biol. 121, 219-227.

Yu, J.G., Furst, D.O., Thornell, L.E., 2003. The mode of myofibril remodelling in human skeletal muscle affected by DOMS induced by eccentric contractions. Histochem. Cell Biol. 119, 383-393. 